\title{
Person-Centered Health Records
}

Zachary King

Electrical and Computer Engineering Department, Purdue School of Engineering and Technology, IUPUI

Current Personal Health Records (PHRs) have been in existence for more than a decade. However, to date, they have not been able to attract the attention of mainstream patients. This limited widespread adoption may possibly be due to a lack of support of the users in efficiently and effectively managing their health records. The exiting PHRs seem to be even less useful to health care providers in support of their decision-making process. Most PHRs include an emergency profile which is entered by the user and can be accessed by emergency care providers. This information is useful and in critical situation may be the only information available to health care providers. However, the PHRs also often rely on the user to enter other health-related information instead of facilitating a user-friendly automated and personalized data integration model with external data sources. User-entered data can make health records lack reliability and render the underlying application difficult to manage for layman users. Furthermore, current PHRs are organized to facilitate data acquisition rather than based on ease of access and of management by mainstream users.

In this paper, we propose a new architecture for PHRs that we believe can overcome the lack of personalization and data integration in current systems thereby leading to a more user-friendly PHR model that may be able to gain active mainstream engagement. The architecture of the proposed PHR is patient-centric and socially-driven. It allows the users to customize their health records and make these records more available to health care providers for tracking, review, and editing. The proposed system will also encourage large scale community engagement and participation in health related research. 